\title{
Quantified Positive Temporal Constraints ${ }^{\star}$
}

\author{
Witold Charatonik and Michał Wrona \\ Institute of Computer Science \\ University of Wrocław
}

\begin{abstract}
A positive temporal template (or a positive temporal constraint language) is a relational structure whose relations can be defined over countable dense linear order without endpoints using a relational symbol $\leq$, logical conjunction and disjunction. This paper gives a complete complexity characterization for quantified constraint satisfaction problems (QCSP) over positive temporal languages. Although the constraint satisfaction problem (CSP) for an arbitrary positive temporal language is trivial (all these templates are closed under constant functions), the corresponding QCSP problems are decidable in LOGSPACE or complete for one of the following classes: NLOGSPACE, P, NP or PSPACE.
\end{abstract}

\section{Introduction}

Constraint Satisfaction Problems provide a uniform approach to research on a wide variety of combinatorial problems. Undisputedly, the most interesting problem in this area is to verify Dichotomy Conjecture posed by Feder and Vardi [1]. It says that every constraint satisfaction problem on a finite domain is either tractable or NP-complete and was inspired by Schaefer's Dichotomy Theorem for CSP on a two element set [2]. When algebraic approach came on the scene the works on dichotomy conjecture were sped up [3]. Although the main goal has not yet been attained, many interesting results were published and many interesting techniques were developed [4,5]. Besides earlier results on constraints over infinite domains [6,7], a new approach was quite recently proposed and developed by Manuel Bodirsky [8] and co-authors. This framework concentrates on relational structures that are $\omega$-categorical. Many results concerning both CSP and QCSP [9] over finite domains were generalized to infinite ones. Moreover, new results were established. Among them there are full characterizations of complexity for both CSP and QCSP of equality constraint languages [10,11].

Our paper is the next step in this research area. In general, we consider quantified constraint satisfaction problems for sets of relations definable over $\langle\mathbb{Q},<\rangle$. In particular, we restrict ourselves to templates definable with $\wedge, \vee$ and $\leq$, i.e., we do not consider negation. We name such relations and languages positive temporal. As in [12], we refer to an arbitrary relation defined over $\langle\mathbb{Q},<\rangle$ as a temporal relation.

Our main contribution is a complexity characterization of QCSP problems over positive temporal languages summarized in Theorem 1 below. We follow the algebraic approach to constraint satisfaction problems: we first classify positive temporal languages depending on their surjective polymorphisms and then give the complexity of QCSP for each obtained class.

\footnotetext{
* Work partially supported by Polish Ministry of Science and Education grant 3 T11C 04230
} 
Theorem 1 (The Main Theorem). Let $\Gamma$ be a language of positive temporal relations, then one of the following holds.

1. Each relation in $\Gamma$ is definable by a conjunction of equations $\left(x_{1}=x_{2}\right)$ and then $Q C S P(\Gamma)$ is decidable in LOGSPACE.

2. Each relation in $\Gamma$ is definable by a conjunction of weak inequalities $\left(x_{1} \leq x_{2}\right)$. If there exists a relation in $\Gamma$ that is not definable as a conjunction of equalities, then $Q C S P(\Gamma)$ is NLOGSPACE-complete.

3. Each relation in $\Gamma$ is definable by a formula of the form $\bigwedge_{i=1}^{n}\left(x_{i_{1}} \leq x_{i_{2}} \vee\right.$ $\left.\ldots \vee x_{i_{1}} \leq x_{i_{k}}\right)$ and then, provided $\Gamma$ satisfies neither condition 1 nor 2 , the set $Q C S P(\Gamma)$ is P-complete.

4. Each relation in $\Gamma$ is definable by a formula of the form $\bigwedge_{i=1}^{n}\left(x_{i_{2}} \leq x_{i_{1}} \vee\right.$ $\left.\ldots \vee x_{i_{k}} \leq x_{i_{1}}\right)$ and then provided $\Gamma$ satisfies neither condition 1 nor 2 , the set $Q C S P(\Gamma)$ is P-complete.

5. Each relation in $\Gamma$ is definable by a formula of the form $\bigwedge_{i=1}^{n}\left(x_{i_{1}}=y_{i_{1}} \vee \ldots \vee\right.$ $\left.x_{i_{k}}=y_{i_{k}}\right)$ and then provided $\Gamma$ does not belong to any of the classes $1-4$, the set $Q C S P(\Gamma)$ is NP-complete.

6. The problem $Q C S P(\Gamma)$ is PSPACE-complete.

Related work. The complete characterization is quite complex and does not fit into one paper. Therefore some parts of Theorem 1 are proved in a companion paper [13]. In particular, we prove there that each QCSP problem over positive temporal relations is either in P or is NP-hard. Here we provide the complete characterization of the NP-hard case, distinguishing between NP-complete and PSPACE-complete cases (items 5 and 6 of Theorem 1). We also give complexity proofs for items 3 and 4 of Theorem 1, leaving the algebraic characterization in [13].

Quantified constraint satisfaction problems over temporal relations were investigated in [11,14]. In particular, it is shown there that quantified problems from item 1 and 2 of Theorem 1 belong to LOGSPACE and NLOGSPACE, respectively. Our result substantially improves these results in the sense that we consider a strictly more expressive class of constraint languages. As in [11] we use the surjective preservation theorem.

The area of CSP may be often seen as a good framework for many problems in AI. In context of our characterization the well-motivated AND/OR precedence constraints [15] should be noted. They are closely related to languages from items 3 and 4 . It might be said that we consider quantified positive variations of AND/OR precedence constraints.

In a very recent paper [12] the authors give a classification of CSP over temporal languages depending on their polymorphisms. Although it sounds similar, it is different from our classification. We deal with positive temporal languages and surjective polymorphisms, which are used to classify QCSP problems (as opposed to CSP problems considered in [12]). In the case of positive temporal languages, the classification based on polymorphisms is trivial: all these languages fall into the same class because they are all closed under constant functions - as a consequence all CSP problems for positive temporal languages are trivial. To obtain our classification we use methods different from those used in [12]. 
Outline of the paper. In Section 2, we give some preliminaries. Among others, we recall a definition of a surjective polymorphism and surjective preservation theorem, which is the most important tool in algebraic approach to QCSP. In [13] it is shown that the problem $\operatorname{QCSP}(\Gamma)$ is NP-hard if and only if $\Gamma$ has essentially unary surjective polymorphisms only. Sections 3 and 4 are devoted to classify positive temporal languages preserved by essentially unary surjections. In Section 3 we show that there are only five different classes of positive temporal relations with different surjective unary polymorphisms. If a positive temporal language $\Gamma$ is closed under all unary surjective polymorphisms, then, as it was shown in [11], each relation of $\Gamma$ may be defined as in item 5 of Theorem 1 and $\operatorname{QCSP}(\Gamma)$ is NP-complete. If a positive temporal language is preserved by some non-trivial subset of unary surjections, then $Q C S P(\Gamma)$ is PSPACEcomplete. We prove it in Section 4. The last section contains a complexity proof for cases 3 and 4 .

\section{Preliminaries}

Relational structures. In most cases we follow the notation from [8, 11]. We consider only relations defined over countable domains and hence whenever we write a domain or $D$ we mean a countable set. Let $\tau$ be some relational (in this paper always finite) signature i.e., a set of relational symbols with assigned arity. Then $\Gamma$ is a $\tau$-structure over domain $D$ if for each relational symbol $R_{i}$ from $\tau$, it contains a relation of according arity defined on $D$. In the rest of the paper we usually say relational language (or pattern) instead of relational structure. Moreover, we use the same notation for relational symbols and relations.

Automorphisms of $\Gamma$ constitute a group with respect to composition. An orbit of a k-tuple $t$ in $\Gamma$ is the set of all tuples of the form $\left\langle\Pi\left(t_{1}\right), \ldots, \Pi\left(t_{k}\right)\right\rangle$ for all automorphisms $\Pi$. We say that a group of automorphisms of $\Gamma$ is oligomorphic if for each $k$ it has a finite number of orbits of $k$-tuples. Although there are many different ways of introducing a concept of $\omega$-categorical structures we do it by the following theorem [16].

Theorem 2. (Engeler, Ryll-Nardzewski, Svenonius) Let $\Gamma$ be a relational structure. Then $\Gamma$ is $\omega$-categorical if and only if the automorphism group of $\Gamma$ is oligomorphic.

Polymorphisms. Let $R$ be a relation of arity $n$ defined over $D$. We say that a function $f$ : $D^{m} \rightarrow D$ is a polymorphism of $R$ if for all $a^{1}, \ldots, a^{m} \in R$ (where $a^{i}$, for $1 \leq i \leq m$, is a tuple $\left.\left\langle a_{1}^{i}, \ldots, a_{n}^{i}\right\rangle\right)$, we have $\left\langle f\left(a_{1}^{1}, \ldots, a_{1}^{m}\right), \ldots, f\left(a_{n}^{1}, \ldots, a_{n}^{m}\right)\right\rangle \in R$. Then we say that $f$ preserves $R$ or that $R$ is closed under $f$. A polymorphism of $\Gamma$ is a function that preserves all relations of $\Gamma$. By $\operatorname{Pol}(\Gamma)$ we denote the set of polymorphisms of $\Gamma$, and by $\operatorname{sPol}(\Gamma)$ - the set of surjective polymorphisms.

An operation $f$ of arity $n$ is essentially unary if there exists a unary operation $f_{0}$ such that $f\left(x_{1}, \ldots, x_{n}\right)=f_{0}\left(x_{i}\right)$ for some fixed $i \in\{1, \ldots, n\}$. An operation that is not essentially unary is called essential.

Quantified constraint satisfaction problems. Let $\Gamma$ contain $R_{1}, \ldots, R_{k}$. Then a conjunctive positive formula (cp-formula) over $\Gamma$ is a formula of the following form:

$$
Q_{1} x_{1} \ldots Q_{n} x_{n}\left(R_{1}\left(\boldsymbol{v}_{\mathbf{1}}\right) \wedge \ldots \wedge R_{k}\left(\boldsymbol{v}_{\boldsymbol{k}}\right)\right)
$$


where $Q_{i} \in\{\forall, \exists\}$ and $\boldsymbol{v}_{\boldsymbol{j}}$ are vectors of variables $x_{1}, \ldots, x_{n}$.

A $\operatorname{QCSP}(\Gamma)$ is a problem to decide whether a given cp-formula without free variables over the structure $\Gamma$ is true or not. Note that by downward Löwenheim-Skolem Theorem we can focus on countable domains only.

If all quantifiers in (1) are existential then such a cp-formula is called positive primitive ( $p p$-formula). A problem to decide whether a given pp-formula over $\Gamma$ is satisfiable is well-known as a constraint satisfaction problem.

A relation $R$ has a cp-definition in $\Gamma$ if there exists a cp-formula $\phi\left(x_{1}, \ldots, x_{n}\right)$ over $\Gamma$ such that for all $a_{1}, \ldots, a_{n}$ we have $R\left(a_{1}, \ldots, a_{n}\right)$ iff $\phi\left(a_{1}, \ldots, a_{n}\right)$ is true. The set of all relations cp-definable in $\Gamma$ is denoted by $[\Gamma]$.

Lemma 1 ([11]). Let $\Gamma_{1}, \Gamma_{2}$ be relational languages. If every relation in $\Gamma_{1}$ has a cpdefinition in $\Gamma_{2}$, then $\operatorname{QCSP}\left(\Gamma_{1}\right)$ is log-space reducible to $\operatorname{QCSP}\left(\Gamma_{2}\right)$.

The following results link $[\Gamma]$ with $\operatorname{sPol}(\Gamma)$. The idea behind Theorem 3 is that the more $\Gamma$ can express, in the sense of cp-definability, the less polymorphisms are contained in $s \operatorname{Pol}(\Gamma)$. Moreover, the converse is also true. This theorem is called surjective preservation theorem.

Theorem 3 ([11]). Let $\Gamma$ be an $\omega$-categorical structure. Then a relation $R$ has a cpdefinition in $\Gamma$ if and only if $R$ is preserved by all surjective polymorphisms of $\Gamma$.

As a direct consequence of Lemma 1 and Theorem 3 we obtain the following.

Corollary 1 ([11]). Let $\Gamma_{1}, \Gamma_{2}$ be w-categorical structures. If $\operatorname{sPol}\left(\Gamma_{2}\right) \subseteq \operatorname{sPol}\left(\Gamma_{1}\right)$, then $\operatorname{QCSP}\left(\Gamma_{1}\right)$ is log-space reducible to $\operatorname{QCSP}\left(\Gamma_{2}\right)$.

Games and cp-definitions. Sometimes it is useful to see a cp-formula $\psi$ without free variables as a two-player game. The game consists of alternating moves of existential and universal player. All variables are evaluated in the order they occur in the quantifier prefix, the existential player evaluates existentially quantified variables and the universal player evaluates universally quantified variables. At the end of the game, the players establish a valuation $q$ from the variables of $\psi$ into the set of rational numbers. We say that one variable is earlier (later) than the another one if it occurs earlier (later) in the quantifier prefix. If at the end of the game, the valuation $q$ satisfies $\psi$, then the existential player wins; otherwise, the universal player is the winner. If the existential player has a winning strategy, then $\psi$ is true; otherwise, if there exists a winning strategy for the universal player, then $\psi$ is false.

Quantified Equality Constraints. Concerning patterns that allow equations and all logical connectives the following classification is known [11].

1. Negative languages. Relations of such a language are definable as CNF-formulas whose clauses are either equalities $(x=y)$ or disjunctions of disequalities $\left(x_{1} \neq\right.$ $\left.y_{1} \vee \ldots \vee x_{k} \neq y_{k}\right)$. For each negative $\Gamma$ the problem $\operatorname{QCSP}(\Gamma)$ is contained in LOGSPACE. 
2. Positive languages. Relations may be defined as a conjunction of disjunctions of equalities $\left(x_{1}=y_{1} \vee \ldots \vee x_{k}=y_{k}\right)$. For each positive $\Gamma$ not being negative the problem $Q \operatorname{CSP}(\Gamma)$ is NP-complete.

3. In any other case the problem $Q \operatorname{CSP}(\Gamma)$ is PSPACE-complete.

Note that the class 1 from Theorem 1 is a subset of Negative languages and the class 5 is just the class of Positive languages.

To give our characterization we need the following result. It may be inferred from lemmas given in Section 7 in [11].

Lemma 2. Let $\Gamma$ be an equational positive constraint language that is preserved by an essential operation on $D$ with infinite image. Then $\Gamma$ is preserved by all operations, and $\Gamma$ is negative.

Corollary 2. If an equational positive constraint language $\Gamma$ is positive, but not negative, then $s$ Pol $(\Gamma)$ contains only essentially unary polymorphisms.

Quantified Positive Temporal Constraints. Now, we focus on positive temporal relations announced in the introduction. All of them are defined over the set of rational numbers using a relational symbol $\leq$ and connectives $\wedge, \vee$. Therefore our results concerning positive temporal relations generalize those for positive equality languages. Since the only relational symbol we use is interpreted as a weak linear order over rational numbers, for each positive temporal structure $\Gamma$ the set $\operatorname{sol}(\Gamma)$ contains all automorphisms that preserve order, i.e., all increasing unary surjections $f: \mathbb{Q} \rightarrow \mathbb{Q}$. Thus, using Theorem 2 , it is not hard to see that all positive temporal languages are $\omega$-categorical.

In [13] we show that each temporal relation is closed not only under all increasing functions but also under all weakly increasing surjections.

\section{Surjective Unary Polymorphisms of Temporal Relations}

This section examines positive temporal relations that are closed only under surjective unary polymorphisms. We want to divide this subset of positive temporal languages into classes each of which contains $\Gamma_{1}$ and $\Gamma_{2}$ if and only if $\operatorname{sPol}\left(\Gamma_{1}\right)=\operatorname{sPol}\left(\Gamma_{2}\right)$ (or equivalently $\left[\Gamma_{1}\right]=\left[\Gamma_{2}\right]$ ). Such a classification facilitates providing complexity results - see Theorem 3.

First we give some preliminary definitions. A permutation of a finite set is a bijection from this set to itself. Let $A=\left\{a_{1}, \ldots, a_{n}\right\}$ be a finite ordered set such that $a_{1}<\ldots<$ $a_{n}$. We say that a permutation $\pi$ of $A$ is a cycle of $A$ if there exists $i \leq n$ such that $\pi\left(a_{i}\right)<\pi\left(a_{i+1}\right)<\ldots<\pi\left(a_{n}\right)<\pi\left(a_{1}\right)<\ldots<\pi\left(a_{i-1}\right)$. Similarly, $\pi$ is a reversed cycle if there exists $i \leq n$ such that $\pi\left(a_{i}\right)>\pi\left(a_{i+1}\right)>\ldots>\pi\left(a_{n}\right)>\pi\left(a_{1}\right)>\ldots>$ $\pi\left(a_{i-1}\right)$.

Definition 1. We say that a relation $R$ is closed under all permutations (respectively, under all cycles or reversed cycles) if for every tuple $\left\langle q_{1}, \ldots, q_{n}\right\rangle \in R$ and every permutation (respectively, every cycle or reversed cycle) $\pi$ of the set $\left\{q_{1}, \ldots, q_{n}\right\}$ we have $\left\langle\pi\left(q_{1}\right), \ldots, \pi\left(q_{n}\right)\right\rangle \in R$. 
Note that in the definition above we permute the set $\left\{q_{1}, \ldots, q_{n}\right\}$ (and not the set of indices $\{1, \ldots, n\}$ ), which may have less then $n$ elements if $q_{1}, \ldots, q_{n}$ are not pairwise distinct.

The preceding definitions concern closure under various kinds of permutations. Although they may look quite similar to closure under polymorphisms, they are different. Below we give some, important for us, examples of (unary) surjective polymorphisms of positive temporal relations. They are all of the type: $\mathbb{Q} \rightarrow \mathbb{Q}$.

Definition 2. We say that a surjection $f: \mathbb{Q} \rightarrow \mathbb{Q}$ is weakly half-increasing (respectively weakly half-decreasing) if there exist two irrational real numbers $x$ and $y$ such that

- $f$ restricted to the set $\{q \in \mathbb{Q} \mid q<x\}$ as well as $f$ restricted to the set $\{q \in \mathbb{Q} \mid$ $q>x\}$ is weakly increasing (respectively, weakly decreasing), and

- for all $q<x$ we have $f(q)>y$ (respectively $f(q)<y$ ) and for all $q>x$ we have $f(q)<y$ (respectively $f(q)>y$ ).

A weakly half-increasing or weakly half-decreasing function is called weakly halfmonotone.

Example 1. Recall that all countable dense linear orders without endpoints are isomorphic. In particular, $\mathbb{Q}$ and $\mathbb{Q} \backslash\{0\}$ are isomorphic, so we may identify $\mathbb{Q}$ with $\mathbb{Q} \backslash\{0\}$ and think of 0 as an irrational number in $\mathbb{Q} \backslash\{0\}$. Then the function $f: \mathbb{Q} \backslash\{0\} \rightarrow \mathbb{Q} \backslash\{0\}$ defined by $f(q)=\frac{1}{q}$ is weakly half-decreasing and the function defined by $f(q)=\frac{-1}{q}$ is weakly half-increasing.

The unary operation $-: \mathbb{Q} \rightarrow \mathbb{Q}$ is defined as $-(x)=-x$ in usual sense.

The rest of this section is devoted to prove the following result.

Theorem 4. Let $\Gamma$ be a set of positive temporal relations such that sPol $(\Gamma)$ contains only essentially unary functions. Then exactly one of the following cases holds.

1. $\operatorname{sPol}(\Gamma)$ is the set of all unary surjections of $\mathbb{Q}$.

2. $\operatorname{sPol}(\Gamma)$ is the set of all weakly increasing, weakly decreasing or weakly halfmonotone surjections of $\mathbb{Q}$.

3. $\operatorname{sPol}(\Gamma)$ is the set of all weakly increasing or weakly decreasing surjections of $\mathbb{Q}$.

4. $\operatorname{sPol}(\Gamma)$ is the set of all weakly increasing or weakly half-increasing surjections of Q.

5. $\operatorname{sPol}(\Gamma)$ is the set of all weakly increasing surjections of $\mathbb{Q}$.

A similar classification considering (not necessarily surjective) unary polymorphisms was obtained in [12]. Weakly half-increasing polymorphisms correspond in some way to the function cyc from that paper. In turn, positive temporal relations preserved by weakly half-decreasing functions correspond to temporal relations closed under - and cyc.

As indicated in Theorem 4, there are only four interesting classes of unary polymorphisms of positive temporal relations: weakly increasing, weakly decreasing, weakly half-increasing, and weakly half-decreasing. The following lemmas say that if some positive temporal relation is closed under one polymorphism of a given class, then it is closed under all polymorphisms of this class. 
Lemma 3. If $s$ Pol $(R)$ contains a weakly decreasing unary surjection $f$, then it contains all weakly decreasing unary surjections.

Lemma 4. If $\operatorname{sPol}(R)$ contains a weakly half-increasing unary surjection $f$, then it contains all weakly half-increasing unary surjections. If sPol $(R)$ contains a weakly half-decreasing unary surjection $f$, then it contains all weakly decreasing, all weakly half-increasing and all weakly half-decreasing unary surjections.

Now, we relate various surjective polymorphisms to closures under various kinds of permutations (see for example Definition 1). In particular, Lemma 5 below implies that the set of positive temporal relations closed under all permutations equals to the set of positive languages from [11].

Lemma 5. A positive temporal relation $R$ is closed under all permutations iff $\operatorname{sol}(R)$ contains all unary surjections of $\mathbb{Q}$.

Lemma 6. A positive temporal relation $R$ is closed under all cycles iff $s P o l(R)$ contains all weakly half-increasing surjections of $\mathbb{Q}$.

Lemma 7. A positive temporal relation $R$ is closed under all reversed cycles iff $\operatorname{siol}(R)$ contains all weakly half-decreasing surjections of $\mathbb{Q}$.

Since we are interested in surjective functions, we can claim the following.

Lemma 8. Let $f$ be a unary, surjective operation on $Q$, then there exist:

- an infinite, strictly monotone sequence $\left(a_{n}\right)_{n \in \mathbb{N}}$ of rational numbers such that $\lim _{n \rightarrow \infty} f\left(a_{n}\right)=+\infty$

- an infinite, strictly monotone sequence $\left(b_{n}\right)_{n \in \mathbb{N}}$ of rational numbers such that $\lim _{n \rightarrow \infty} f\left(b_{n}\right)=-\infty$

To prove Theorem 4, we show that if $\operatorname{sPol}(\Gamma)$ contains any function that is neither weakly monotone nor weakly half-monotone, then it contains all unary rational functions or equivalently, by Lemma 5, is closed under all permutations.

Lemma 9. Let $R$ be a positive temporal relation such that sPol $(R)$ contains a function $f$ that is neither weakly increasing nor weakly decreasing nor weakly half-monotone. Let $\left(c_{n}\right)_{n \in \mathbb{N}}$ and $\left(d_{n}\right)_{n \in \mathbb{N}}$ be two strictly monotone sequences satisfying the following: $\lim _{n \rightarrow \infty} f\left(c_{n}\right)=+\infty$ and $\lim _{n \rightarrow \infty} f\left(d_{n}\right)=-\infty$. Then $R$ is closed under all permutations.

Proof. (of Theorem 4) Suppose that $s \operatorname{Pol}(\Gamma)$ contains only essentially unary functions. If $s P o l(\Gamma)$ contains a function $f$ that is neither weakly monotone nor weakly half-monotone, then by Lemma 8 we find two strictly monotone sequences $\left(a_{n}\right)_{n \in \mathbb{N}}$ and $\left(b_{n}\right)_{n \in \mathbb{N}}$ such that $\lim _{n \rightarrow \infty} f\left(a_{n}\right)=+\infty$ and $\lim _{n \rightarrow \infty} f\left(b_{n}\right)=-\infty$. Then by Lemma 9 every relation in $\Gamma$ is closed under all permutations, so by Lemma $5 \operatorname{sPol}(R)$ contains all essentially unary surjections. Hence $\operatorname{sPol}(\Gamma)$ is the set of all essentially unary surjections of $\mathbb{Q}$ and we are in case 1 . 
Now assume that $\operatorname{sol}(\Gamma)$ contains only weakly monotone or weakly half-monotone surjections. There are four cases, depending on whether $\operatorname{sPol}(\Gamma)$ contains a weakly decreasing surjection or a weakly half-increasing surjection.

If $\operatorname{sPol}(\Gamma)$ contains a weakly decreasing surjection and a weakly half-increasing surjection, then it contains a weakly half-decreasing surjection and by Lemmas 3 and 4, it contains all weakly decreasing and all weakly half-monotone surjections of $\mathbb{Q}$, so we are in case 2.

If $\operatorname{sol}(\Gamma)$ contains a weakly decreasing surjection and it does not contain any weakly half-increasing surjection, then by lemmas 3 and 4 it contains all weakly decreasing and it does not contain any weakly half-monotone surjections, so we are in case 3.

If $\operatorname{sPol}(\Gamma)$ does not contain any weakly decreasing surjection and it contains a weakly half-increasing surjection, then by Lemma 4 it contains all weakly half-increasing and it does not contain weakly decreasing surjections, so we are in case 4 .

Finally, if $s P o l(\Gamma)$ does not contain any weakly decreasing surjection and it does not contain any weakly half-increasing surjection, then by Lemma 4 it does not contain any weakly decreasing nor weakly half-monotone surjection, so we are in case 5.

Example 2. Recall from [11] that $\left(x_{1}=x_{2} \vee x_{1}=x_{3}\right)$ is closed under all essentially unary surjections of $\mathbb{Q}$ - see also Section 2. Now, for each of the classes 2-5 of Theorem 4 we give representatives, that is, relations $R_{(5)}-R_{(2)}$ each of which belongs to exactly one of these classes.

The relation $R_{(5)}$ defined by $R_{(5)}\left(x_{1}, x_{2}, x_{3}\right):=\left(x_{1} \leq x_{2} \vee x_{2} \leq x_{3}\right)$, as all positive temporal relations, is closed under all weakly increasing functions. Observe that $\langle 1,2,3\rangle \in R_{(5)}$, but $\langle-1,-2,-3\rangle \notin R_{(5)}$, so $R_{(5)}$ is not closed under weakly decreasing functions (and by Lemma 4 it is not closed under half-decreasing functions). Similarly, $\langle 1,3,2\rangle \in R_{(5)}$, but $\langle 3,2,1\rangle \notin R_{(5)}$, so $R_{(5)}$ is not closed under cycles (and thus it is not closed under weakly half-increasing functions). The relation $R_{(4)}$ defined by $R_{(4)}\left(x_{1}, x_{2}, x_{3}\right):=\left(x_{1} \leq x_{2} \vee x_{2} \leq x_{3}\right) \wedge\left(x_{2} \leq x_{3} \vee x_{3} \leq x_{1}\right) \wedge\left(x_{3} \leq\right.$ $\left.x_{1} \vee x_{1} \leq x_{2}\right)$ is a conjunction of the relations $\left(x_{\Pi(1)} \leq x_{\Pi(2)} \vee x_{\Pi(2)} \leq x_{\Pi(3)}\right)$ where $\Pi$ ranges over all cycles of the set $\{1,2,3\}$, so it is closed under all cycles. Since $\langle 1,2,3\rangle \in R_{(4)}$ and $\langle 3,2,1\rangle \notin R_{(4)}$, it is not closed under weakly decreasing or weakly half-decreasing functions. It is easy to observe that the relation $R_{(3)}$ defined by $R_{(3)}\left(x_{1}, x_{2}, x_{3}\right):=\left(x_{1} \leq x_{2} \vee x_{2} \leq x_{3}\right) \wedge\left(x_{3} \leq x_{2} \vee x_{2} \leq x_{1}\right)$ is closed under weakly decreasing functions. Since $\langle 2,1,3\rangle \in R_{(3)}$ and $\langle 3,2,1\rangle \notin R_{(3)}$, this relation is not closed under cycles and by Lemmas 6 and 4 it is not closed under any weakly half-monotone surjection. Let a relation $R_{(2)}$ be defined as a conjunction of the clauses $\left(x_{\Pi(1)} \leq x_{\Pi(2)} \vee x_{\Pi(2)} \leq x_{\Pi(3)} \vee x_{\Pi(3)} \leq x_{\Pi(4)}\right)$ where $\Pi$ ranges over all cycles and reversed cycles of the set $\{1,2,3,4\}$, so it obviously must be closed under all cycles and reversed cycles. Note that cycles and reversed cycles are 8 out of total 24 permutations of the set $\{1,2,3,4\}$ (this explains why we could not use a ternary relation as an example here - all permutations of the set $\{1,2,3\}$ are either cycles or reversed cycles). To see that $R_{(2)}$ is not closed under all permutations observe that $\langle 4,3,2,1\rangle \notin R_{(2)}$, but $\langle 2,1,3,4\rangle \in R_{(2)}$.

Finally, we show that all these relations $\left(R_{(2)}-R_{(5)}\right)$ are closed under essentially unary surjections only. Let $R\left(x_{1}, \ldots, x_{k}\right)$ where $k=3,4$ be one of these relations. 
Then a relation $\bigwedge_{\Pi \in S_{k}} R\left(x_{\Pi(1)}, \ldots, x_{\Pi(k)}\right)$ where $S_{k}$ is a set of all permutations on $k$ elements is equivalent to a relation $R^{\prime}$ defined by $\bigvee_{i \neq j} x_{i}=x_{j}$. Because $R^{\prime}$ is positive and non-negative, by Corollary 2 and Theorem 3, we have that $R$ is closed under unary surjections only.

\section{PSPACE-complete Positive Temporal Languages}

Recall from Section 2 the complexity characterization of equational languages. By corollaries 2 and 1, the problem $\operatorname{QCSP}(\Gamma)$ where $\Gamma$ is closed under essentially unary functions only is NP-hard. Likewise we know that QCSP for languages from item 1 of Theorem 4 is NP-complete. This section is devoted to show PSPACE-completeness for QCSP of languages with surjective polymorphisms from items 2-5 of Theorem 4.

Membership in PSPACE is the simpler part of the proof and is common for all, not only positive, temporal relations.

Proposition 1. For every temporal language $\Gamma$, the problem $Q \operatorname{CSP}(\Gamma)$ is decidable in PSPACE.

In the rest of the section we prove hardness. Note that the set of surjective polymorphisms from item 2 contains sets of surjective polymorphisms from each of items 2-5. Therefore, by Theorem 3 and Corollary 1, it is enough to show PSPACE-hardness of QCSP for positive temporal languages closed under all weakly monotone and all weakly half-monotone surjections only.

Theorem 5. Let $\Gamma$ be a set of positive languages closed only under essentially unary functions. If $\operatorname{sPol}(\Gamma)$ is the set of all weakly increasing, weakly decreasing and weakly half-monotone surjections of $\mathbb{Q}$, then $Q C S P(\Gamma)$ is PSPACE-hard.

Because of Theorem 3 and Corollary 1, it is enough to choose just one language with appropriate set of surjective polymorphisms and show PSPACE hardness for this language. Our choice is the language $\Gamma_{\text {Circle }}$ defined below. We show that it is closed only under all weakly increasing, weakly decreasing and weakly half-monotone surjections of $\mathbb{Q}$. In fact, it is enough to show that $\Gamma_{\text {circle }}$ is closed only under unary surjections of $\mathbb{Q}$ and that is closed under all reversed cycles - see lemmas 4 and 7. Finally, we show that $Q \operatorname{CSP}\left(\Gamma_{\text {Circle }}\right)$ is PSPACE-hard and in consequence we prove Theorem 5.

Definition of $\Gamma_{\text {Circle. }}$. First we present some auxiliary relations that shorten the definition. Let $\boldsymbol{v}_{A}$ be a vector $\left\langle B_{0}, O_{0}^{L}, I_{0}^{L}, I_{0}^{R}, O_{0}^{R}, B_{1}, O_{1}^{L}, I_{1}^{L}, I_{1}^{R}, O_{1}^{R}, B_{2}\right\rangle$ of variables ranging over $\mathbb{Q}$. The corresponding set of variables is denoted by $\operatorname{Var}_{A}$. In the following we call $\operatorname{Var}_{A}$ the set of arena variables. We sometimes see a vector $\boldsymbol{v}_{\boldsymbol{A}}$ as a function from $\{0, \ldots, 10\}$ to $\operatorname{Var}_{A}$.

Let Arenas be a set of vectors $\Pi\left(\boldsymbol{v}_{\boldsymbol{A}}\right)$ for all cycles and reversed-cycles $\Pi$ of the set $\{0, \ldots, 10\}$. Note that the set Arenas may be represented in some way using Fig. 1. To obtain one of linear orders that is represented by this circle, we have just to tear it apart and orientate. If we orientate it clockwise, then we represent some $\Pi_{C}\left(\boldsymbol{v}_{\boldsymbol{A}}\right)$ where $\Pi_{C}$ is a cycle. Otherwise, if we orientate it anticlockwise, then we represent some $\Pi_{R C}\left(\boldsymbol{v}_{\boldsymbol{A}}\right)$ where $\Pi_{R C}$ is a reversed cycle. 


$\begin{array}{llll} & I_{0}^{L} & I_{0}^{R} & \\ O_{0}^{L} & & & O_{0}^{R}\end{array}$

$B_{0}$

$B_{1}$

$B_{2}$

$\begin{array}{llll} & & & O_{1}^{L} \\ & & & \\ & I_{1}^{R} & I_{1}^{L} & \end{array}$

Fig. 1. The representation of the set Arenas

Now, for each $\boldsymbol{v} \in$ Arenas we define a relation Prefix $\boldsymbol{v}:=\neg\left(y_{0}<\ldots<y_{10}\right)$ where $\boldsymbol{v}=\left\langle y_{0}, \ldots, y_{10}\right\rangle$. At this point we probably owe the reader one more explanation. Sometimes, when we think it is intuitive, we use negation as well as implication in the definition of relations. Nevertheless, they should be treated just as notational shortcuts and all relations we claim to be positive temporal are indeed definable by conjunction, disjunction, and $\leq$. In particular, Prefix $\boldsymbol{v}_{\boldsymbol{v}}$ may be defined as $\bigvee_{i=0}^{9} y_{i} \geq y_{i+1}$. Nevertheless, the situation where Prefix $v_{v}$ is falsified is more important for us. Then the arena variables are arranged in some linear order represented by a circle in Fig. 1.

In general, our intention is to model (see Definition 3) a boolean relation. Arena variables set in some order presented in Fig. 1 constitute some kind of arena. When some other variable is set strictly between $O_{0}^{L}$ and $O_{0}^{R}$ then we see its value as a boolean zero, and if some variable is set strictly between $O_{1}^{L}$ and $O_{1}^{R}$ then we see its value as a boolean one. We need also $I_{0}^{L}, I_{0}^{R}$, and $I_{1}^{L}, I_{1}^{R}$. Sometimes we want to say: 'If a variable $x$ is equal to zero, then a variable $y$ is also equal to zero'. Unfortunately, concerning positive temporal relations we are unable to write something like $\left(O_{0}^{L}<x<O_{0}^{R}\right) \rightarrow$ $\left(O_{0}^{L}<y<O_{0}^{R}\right)$. Instead we write $\left(O_{0}^{L}<x<O_{0}^{R}\right) \rightarrow\left(I_{0}^{L} \leq y \leq I_{0}^{R}\right)$ and assure that $I_{0}^{L}, I_{0}^{R}$ are always strictly between $O_{0}^{L}, O_{0}^{R}$. Similarly, we assure that $I_{1}^{L}, I_{1}^{R}$ are always strictly between $O_{1}^{L}, O_{1}^{R}$. This is the general idea, but sometimes because of technical reasons we also use $O_{0}^{L 1}, O_{0}^{L 2}$ etc.

Concerning positive temporal relations closed under reversed cycles it is hard to say that some variable must be set on the left (or on the right) of the another variable. Far more natural is to say that some variable is inside the interval set by values of other variables or outside such an interval. We define $\operatorname{In}_{\boldsymbol{v}}\left(x, y_{1}, y_{2}\right)$ equal to $\left(\left(y_{1}<y_{2}\right) \rightarrow\right.$ $\left.\left(y_{1} \leq x \leq y_{2}\right)\right) \wedge\left(\left(y_{2}<y_{1}\right) \rightarrow\left(x \leq y_{2} \vee x \geq y_{1}\right)\right)$ if $\boldsymbol{v}=\Pi_{C}\left(\boldsymbol{v}_{\boldsymbol{A}}\right)$ for some cycle $\Pi_{C}$; and equal to $\left(\left(y_{1}<y_{2}\right) \rightarrow\left(x \leq y_{1} \vee x \geq y_{2}\right)\right) \wedge\left(\left(y_{2}<y_{1}\right) \rightarrow\left(y_{2} \leq x \leq y_{1}\right)\right)$ if $\boldsymbol{v}=\Pi_{R C}\left(\boldsymbol{v}_{\boldsymbol{A}}\right)$ for some reversed-cycle $\Pi_{R C}$. Similarly, we define Out $_{\boldsymbol{v}}\left(x, y_{1}, y_{2}\right)$ equal to $\left(\left(y_{1}<y_{2}\right) \rightarrow\left(x \leq y_{1} \vee x \geq y_{2}\right)\right) \wedge\left(\left(y_{2}<y_{1}\right) \rightarrow\left(y_{2} \leq x \leq y_{1}\right)\right)$ if $\boldsymbol{v}=\Pi_{C}\left(\boldsymbol{v}_{\boldsymbol{A}}\right)$ for some cycle $\Pi_{C}$; and equal to $\left(\left(y_{1}<y_{2}\right) \rightarrow\left(y_{1} \leq x \leq y_{2}\right)\right) \wedge\left(\left(y_{2}<\right.\right.$ $\left.\left.y_{1}\right) \rightarrow\left(x \leq y_{2} \vee x \geq y_{1}\right)\right)$ if $\boldsymbol{v}=\Pi_{R C}\left(\boldsymbol{v}_{\boldsymbol{A}}\right)$ for some reversed-cycle $\Pi_{R C}$. 
Example 3. For every $\boldsymbol{v} \in$ Arenas the following formulas are always true:

1. $\left(\neg P r e f i x_{\boldsymbol{v}}\right) \rightarrow \operatorname{In}_{\boldsymbol{v}}\left(I_{0}^{R}, O_{0}^{R}, O_{0}^{L}\right)$

2. $\left(\neg \operatorname{Prefix}_{\boldsymbol{v}}\right) \rightarrow \operatorname{Out}_{\boldsymbol{v}}\left(O_{0}^{R}, I_{0}^{R}, I_{0}^{L}\right)$

The positive temporal language $\Gamma_{\text {Circle }}$ consists of three relations: UImp, BImp, and Final. Each relation $R \in \Gamma_{\text {Circle }}$ is of the form $\bigwedge_{\boldsymbol{v} \in \text { Arenas }} \phi_{v}^{R}$. By using this conjunction we assure that $R$ is closed under all cycles and reversed cycles.

1. First of our relations is $\operatorname{UImp}\left(\boldsymbol{v}_{\boldsymbol{A}}, p, O^{L}, O^{R}, f, I^{L}, I^{R}\right)$ with

$$
\phi_{\boldsymbol{v}}^{\text {UImp }}:=\operatorname{Prefix}_{\boldsymbol{v}} \vee \operatorname{Out}_{\boldsymbol{v}}\left(p, O^{L}, O^{R}\right) \vee \operatorname{In}_{\boldsymbol{v}}\left(f, I^{L}, I^{R}\right) .
$$

The name UImp stands for unary implication. It is justified by the context in which we use it. If both Prefix $\boldsymbol{v}_{\boldsymbol{v}}$ and $\operatorname{Out}_{\boldsymbol{v}}\left(p, O^{L}, O^{R}\right)$ are falsified, then $\operatorname{In}_{\boldsymbol{v}}\left(f, I^{L}, I^{R}\right)$ must be satisfied. We use this relation to express the implication: 'if $v$ represents different values in an appropriate order and $p$ is a value in the interval from $I^{L}$ to $I^{R}$, then $f$ also is a value in this interval'.

2. We have also binary implication $\operatorname{BImp}\left(\boldsymbol{v}_{\boldsymbol{A}}, p_{1}, p_{2}, O^{L}, O^{R}, f, I^{L}, I^{R}\right)$ with

$$
\phi_{\boldsymbol{v}}^{B I m p}:=\operatorname{Prefix}_{\boldsymbol{v}} \vee \operatorname{Out}_{\boldsymbol{v}}\left(p_{1}, O^{L}, O^{R}\right) \vee O u t_{\boldsymbol{v}}\left(p_{2}, O^{L}, O^{R}\right) \vee \operatorname{In}_{\boldsymbol{v}}\left(f, I^{L}, I^{R}\right) .
$$

If Prefix $\boldsymbol{v}_{\boldsymbol{v}}$ as well as $\operatorname{Out}_{\boldsymbol{v}}\left(p_{1}, O^{L}, O^{R}\right)$ and $\operatorname{Out}_{\boldsymbol{v}}\left(p_{2}, O^{L}, O^{R}\right)$ are falsified, then $\operatorname{In}_{\boldsymbol{v}}\left(f, I^{L}, I^{R}\right)$ must be satisfied.

3. Finally there is Final $\left(\boldsymbol{v}_{\boldsymbol{A}}, f_{0}, f_{1}\right)$ with

$$
\phi_{\boldsymbol{v}}^{\text {Final }}:=\operatorname{Prefix}_{\boldsymbol{v}} \vee \operatorname{Out}_{\boldsymbol{v}}\left(f_{0}, B_{0}, B_{2}\right) \vee \operatorname{Out}_{\boldsymbol{v}}\left(f_{1}, B_{0}, B_{2}\right) .
$$

We want to see it in the following way. If Prefix $\boldsymbol{v}$ is falsified, then $\operatorname{Out}_{\boldsymbol{v}}\left(f_{0}, B_{0}, B_{2}\right)$ or $\operatorname{Out}_{\boldsymbol{v}}\left(f_{1}, B_{0}, B_{2}\right)$ must be satisfied.

Lemma 10. The positive temporal language $\Gamma_{\text {Circle }}$ is closed under weakly increasing, weakly decreasing, and weakly half monotone surjections only.

PSPACE-hardness of $Q C S P\left(\Gamma_{\text {Circle }}\right)$. The hardness proof for $Q \operatorname{CSP}\left(\Gamma_{\text {Circle }}\right)$ is based on the proof of PSPACE-hardness of $\operatorname{QCSP}\left(x_{1} \neq x_{2} \vee x_{1}=x_{3}\right)$ from [11]. We define analogous notions and follow analogous reasoning.

Definition 3. A relation $R \subseteq\{0,1\}^{n}$ is force definable if there exists a prenex formula

$$
\Phi_{R, f_{0}, f_{1}}\left(\boldsymbol{v}_{\boldsymbol{A}}, O_{0}^{L 1}, O_{0}^{R 1}, O_{1}^{L 1}, O_{1}^{R 1}, x_{1}, \ldots, x_{n}\right)=\mathcal{Q} \phi
$$

over $\Gamma_{\text {Circle }}$ that satisfies all of the following.

1. $\mathcal{Q}$ is a quantifier prefix and $\phi$ is a quantifier-free part.

2. The quantifier prefix $\mathcal{Q}$ contains $f_{0}$ and $f_{1}$ as its two last variables, and they are both existentially quantified.

3. The set of free variables is equal to $\left\{\boldsymbol{v}_{\boldsymbol{A}}, O_{0}^{L 1}, O_{0}^{R 1}, O_{1}^{L 1}, O_{1}^{R 1}, x_{1}, \ldots, x_{n}\right\}$.

4. Let $\boldsymbol{t} \in\{0,1\}^{n}$ and let $\boldsymbol{v} \in$ Arenas. Let variables from $\operatorname{Var}_{A}$ be set to satisfy $\neg($ Prefix $\boldsymbol{v})$ and let variables $O_{0}^{L 1}, O_{0}^{R 1}, O_{1}^{L 1}, O_{1}^{R 1}$ be set to satisfy 
- $\operatorname{In}_{\boldsymbol{v}}\left(O_{0}^{L 1}, B_{0}, O_{0}^{L}\right)$,

- $\operatorname{In}_{\boldsymbol{v}}\left(O_{0}^{R 1}, O_{0}^{R}, B_{1}\right)$,

- $\operatorname{In}_{\boldsymbol{v}}\left(O_{1}^{L 1}, B_{1}, O_{1}^{L}\right)$, and

- $\operatorname{In}_{\boldsymbol{v}}\left(O_{1}^{R 1}, O_{1}^{R}, B_{2}\right)$.

Further, let $x_{k}$ for $k \in\{1, \ldots, n\}$ are set to satisfy $\operatorname{In}_{\boldsymbol{v}}\left(x_{k}, I_{i}^{L}, I_{i}^{R}\right)$ iff $\boldsymbol{t}_{k}=i$ for $i=0,1$. Then the sentence $\Phi^{\prime}:=\mathcal{Q}\left(\phi \wedge \neg\left(\operatorname{In}_{\boldsymbol{v}}\left(f_{0}, I_{0}^{L}, I_{0}^{R}\right) \wedge \operatorname{In}_{\boldsymbol{v}}\left(f_{1}, I_{1}^{L}, I_{1}^{R}\right)\right)\right)$ is false iff $\boldsymbol{t} \in R$.

5. If values of arena variables satisfy Prefix $\boldsymbol{v}_{\boldsymbol{v}}$ for all $\boldsymbol{v} \in$ Arenas; or, in case Prefix $\boldsymbol{v}$ is falsified for some $\boldsymbol{v} \in$ Arenas, free variables $O_{0}^{L 1}, O_{0}^{R 1}, O_{1}^{L 1}, O_{1}^{R 1}$ are set to satisfy

- $\operatorname{In}_{\boldsymbol{v}}\left(O_{0}^{L 1}, I_{0}^{L}, I_{0}^{R}\right) \wedge \operatorname{In}_{\boldsymbol{v}}\left(O_{0}^{R 1}, I_{0}^{L}, I_{0}^{R}\right) \vee$

- $\operatorname{In}_{\boldsymbol{v}}\left(O_{1}^{L 1}, I_{1}^{L}, I_{1}^{R}\right) \wedge \operatorname{In}_{\boldsymbol{v}}\left(O_{1}^{R 1}, I_{1}^{L}, I_{1}^{R}\right)$;

then $\Phi^{\prime}$ is always true.

6. (monotonicity) For any setting to the free variables of $\Phi_{R, f_{0}, f_{1}}$, if the formula $\Phi^{\prime}$ is true, then changing the value of any variable $x_{i}$ to satisfy $\left(\right.$ Out $_{\boldsymbol{v}}\left(x_{i}, O_{0}^{L 1}, O_{0}^{R 1}\right) \mathrm{V}$ Out $\left.\boldsymbol{v}_{\boldsymbol{v}}\left(x_{i}, O_{1}^{L 1}, O_{1}^{R 1}\right)\right)$ preserves the truth of $\Phi^{\prime}$.

As it was described in Section 2 we can see a sentence as a two-player game. The intuition behind Definition 3 is as follows. If free variables of $\Phi_{R, f_{0}, f_{1}}$ are set according to conditions from item 4 and $t \in R$, then the universal player has a strategy to force the existential player to satisfy $\operatorname{In}_{\boldsymbol{v}}\left(f_{0}, I_{0}^{L}, I_{0}^{R}\right)$ and $\operatorname{In}_{\boldsymbol{v}}\left(f_{1}, I_{1}^{L}, I_{1}^{R}\right)$ where $\boldsymbol{v} \in$ Arenas and Prefix $\boldsymbol{v}_{\boldsymbol{v}}$ is falsified. But if Prefix $\boldsymbol{v}_{\boldsymbol{v}}$ is falsified for some $\boldsymbol{v} \in$ Arenas and the condition from item 5 is fulfilled, then the existential player is able to falsify $\operatorname{In}_{\boldsymbol{v}}\left(f_{0}, I_{0}^{L}, I_{0}^{R}\right)$ or $\operatorname{In}_{\boldsymbol{v}}\left(f_{1}, I_{1}^{L}, I_{1}^{R}\right)$.

Note that variables $O_{0}^{L 1}, O_{0}^{R 1}, O_{1}^{L 1}, O_{1}^{R 1}$ are different from $O_{0}^{L}, O_{0}^{R}, O_{1}^{L}, O_{1}^{R}$.

Lemma 11. There exists a polynomial-time algorithm that, given a boolean circuit $C$ as input, produces a force definition of the relation $R_{C}$ containing, as tuples, exactly the satisfying assignments of the circuit.

Similarly as in [11], we reduce from succinct graph unreachability. In this problem, the input is a boolean circuit with $2 c$ inputs that represent a graph $G$ whose vertices are the tuples in $\{0,1\}^{c}$. There is a directed edge $(\boldsymbol{X}, \boldsymbol{Y})$ in the graph iff the circuit returns true given the input $(\boldsymbol{X}, \boldsymbol{Y})$. The question is to decide whether or not there is a directed path from $\boldsymbol{S}$ to $\boldsymbol{T}$. This problem is known to be PSPACE-complete.

Define $R_{i} \subseteq\{0,1\}^{2 c}$ to be the relation containing exactly the tuples $(\boldsymbol{X}, \boldsymbol{Y})$ such that there exists a directed path in $G$ from $\boldsymbol{X}$ to $\boldsymbol{Y}$ of length less than or equal to $2^{i}$. Then there is a path in $G$ from $\boldsymbol{S}$ to $\boldsymbol{T}$ iff $(\boldsymbol{S}, \boldsymbol{T}) \in R_{c}$.

From Lemma 11 it is not hard to infer that $R_{0}$ is computable in polynomial time. Now, by induction we show that $R_{c}$ is also computable by a polynomial algorithm.

Lemma 12. The force definition of the relation $R_{c}$ is computable in polynomial time.

Proof. (of Theorem 5) Let $\Phi_{R_{c}, g_{0}, g_{1}}\left(\boldsymbol{v}_{\boldsymbol{A}}, O_{0}^{L}, O_{0}^{R}, O_{1}^{L}, O_{1}^{R}, \boldsymbol{x}, \boldsymbol{y}\right)=Q_{c} \phi_{c}$ be a force definition of $R_{c}$. We use it now to give an instance of $\operatorname{QCSP}\left(\Gamma_{\text {Circle }}\right)$ that is true if and only if there is no path from $\boldsymbol{S}$ to $\boldsymbol{T}$ in the succinctly represented graph.

The instance created is 


$$
\forall \boldsymbol{v}_{\boldsymbol{A}} Q_{c} \phi_{c} \wedge \boldsymbol{x}=\boldsymbol{s} \wedge \boldsymbol{y}=\boldsymbol{t} \wedge \operatorname{Final}\left(\boldsymbol{v}_{\boldsymbol{A}}, g_{0}, g_{1}\right)
$$

where $\boldsymbol{s}_{i}=\boldsymbol{t}_{j}=I_{k}^{L}$ if $\boldsymbol{S}_{i}=\boldsymbol{T}_{j}=k$ for all $1 \leq i, j \leq c$ and $k=0,1$.

The universal player starts the game. To have a chance to win (to falsify) the sentence he must set arena variables to falsify Prefix for some $\boldsymbol{v} \in$ Arenas. Otherwise each clause from $\phi_{c}$ is satisfied already at the beginning. If there is a path from $s$ to $t$, then the universal player can enforce the existential player to satisfy $\operatorname{In}_{\boldsymbol{v}}\left(g_{0}, I_{0}^{L}, I_{0}^{R}\right)$ and $\operatorname{In}_{\boldsymbol{v}}\left(g_{1}, I_{1}^{L}, I_{1}^{R}\right)$. It is not hard to verify that it contradicts Final $\left(\boldsymbol{v}_{\boldsymbol{A}}, g_{0}, g_{1}\right)-$ see (4). If $(s, t) \notin R_{c}$, then the existential player can satisfy $\operatorname{Out}_{\boldsymbol{v}}\left(g_{0}, B_{0}, B_{2}\right)$ or $\operatorname{Out}_{\boldsymbol{v}}\left(g_{1}, B_{0}, B_{2}\right)$; and in consequence satisfy Final $\left(\boldsymbol{v}_{\boldsymbol{A}}, g_{0}, g_{1}\right)$.

\section{PTIME-complete Positive Temporal Languages}

This section is devoted to give the complexity proof for classes from items 3 and 4 of Theorem 1 . We focus here of the former case, the latter one is dual and hence the whole reasoning is similar in both cases.

Let $R_{L e f t}^{k}$ be equal to $\left(x_{1} \leq x_{2} \vee \ldots \vee x_{1} \leq x_{k}\right)$, and $\Gamma_{\text {Left }}$ be the set of relations $R_{\text {Left }}^{k}$ for each natural number $k \geq 2$. In [13] it is shown that $Q C S P\left(\Gamma_{\text {Left }}\right)$ is log-space equivalent to $\operatorname{QCSP}(\Gamma)$ where $\Gamma$ is the language from case 3 of Theorem 1. Furthermore, we have that $Q \operatorname{CSP}\left(x_{1} \leq x_{2} \vee x_{1} \leq x_{2}\right)$ is $\log$-space equivalent to $Q C S P\left(\Gamma_{\text {Left }}\right)$. Observe that the definition of each $R_{\text {Left }}^{k}$ have a simple tree-like structure where $x_{1}$ is a root and $x_{2}, \ldots, x_{k}$ are sons of $x_{1}$. Moreover, to denote a root of a clause $C$ we write $\operatorname{root}(C)$ and to denote a set of sons $-\operatorname{sons}(C)$.

In this section whenever we write: a formula, we think of a cp-formula over $\Gamma_{\text {Left }}$.

Lemma 13. Let $\phi$ be a formula defining a positive temporal relation. Assume that $\phi$ contains clauses $C_{1}:=\left(y \leq x_{1} \vee \ldots \vee y \leq x_{k}\right)$ and $C_{2}:=\left(x_{1} \leq z_{1} \vee \ldots \vee x_{1} \leq z_{l}\right)$. Then $\phi$ and $\phi^{\prime}$ given by $\phi \wedge C_{3}$, where $C_{3}:=\left(y \leq z_{1} \vee \ldots \vee y \leq z_{l} \vee y \leq x_{2} \vee \ldots \vee y \leq\right.$ $\left.x_{k}\right)$, are equivalent, that is, they define the same relation.

In the following, we sometimes refer to a quantifier-free formula $\phi$ as to a set of clauses. We say that a set of clauses $\phi$ is TClosed if for all pairs of clauses of the form $\left(y \leq x_{1} \vee \ldots \vee y \leq x_{k}\right)$ and $\left(x_{1} \leq z_{1} \vee \ldots \vee x_{1} \leq z_{l}\right)$, the clause $\left(y \leq z_{1} \vee \ldots \vee y \leq\right.$ $z_{l} \vee y \leq x_{2} \vee \ldots y \vee \leq x_{k}$ ) also belongs to $\phi$. By TClosure $(\phi)$ we denote the least TClosed superset of $\phi$.

By a simple induction, from Lemma 13 we can obtain the following.

Corollary 3. Formulas $\phi$ and TClosure $(\phi)$ are equivalent.

We show that the universal player has a winning strategy if and only if TClosure $(\phi)$ contains a clause of the form $\left(y \leq x_{1} \vee \ldots \vee y \leq x_{k}\right)$ such that for each disjunct $y \leq x_{i}$ where $1 \leq i \leq k$ we have that either $y$ or $x_{i}$ is later and universal. We call such a clause ultimate.

Lemma 14. Let $\psi$ be a sentence and let $Q$ be its quantifier prefix and $\phi$ its quantifierfree part. Then $\psi$ is false if and only if TClosure $(\phi)$ contains an ultimate clause. 
To show the exact complexity of case 3 of Theorem 1 we use the emptiness problem for context-free grammars. It is well known that this problem is P-complete. We assume that the reader is familiar with the notion of the context-free grammar. By $\mathcal{L}(G)$ we denote a language generated by a context free-grammar $G=\langle N, \Sigma, R, S\rangle$.

Theorem 6. Let $\Gamma$ be a positive temporal language such that each of its relation is definable by a formula of the form $\bigwedge_{i=1}^{n}\left(x_{i_{1}} \leq x_{i_{2}} \vee \ldots \vee x_{i_{1}} \leq x_{i_{k}}\right)$ and it is neither definable as a conjunction of equalities nor as a conjunction of inequalities. Then the problem $Q C S P(\Gamma)$ is $P$-complete

Proof. (About Membership) To obtain the result we give a logspace reduction from the problem $\operatorname{QCSP}\left(x_{1} \leq x_{2} \vee x_{1} \leq x_{3}\right)$ to the emptiness problem for context-free grammars. Let $\psi$ be an instance of $\operatorname{QCSP}\left(x_{1} \leq x_{2} \vee x_{1} \leq x_{3}\right)$ with a quantifier prefix $Q$ and a quantifier free-part $\phi$. We construct a context-free grammar $G_{\psi}$ such that $\mathcal{L}\left(G_{\psi}\right) \neq \emptyset$ if and only if $\psi$ is false. By Lemma 14, it is enough to show $G_{\psi}$ that generates a non-empty language if and only if $T C l o s u r e(\phi)$ contains an ultimate clause.

The reduction runs as follows. For each variable $x$ of $\psi$, we show a grammar $G_{\psi}^{x}$ that generates an empty language if and only if there is no ultimate clause $C$ in TClosure $(\phi)$ with $x$ being a root of $C$. Further, by $G_{\psi}$ we take a grammar such that $\mathcal{L}\left(G_{\psi}\right)=$ $\bigcup_{x \in \operatorname{Var}(\psi)} \mathcal{L}\left(G_{\psi}^{x}\right)$ where $\operatorname{Var}(\psi)$ is a set of all variables of $\psi$. Recall that the set of context-free grammars is closed under union and note that $\left\|G_{\psi}\right\| \leq c * \Sigma_{x \in \operatorname{Var}(\psi)}\left\|G_{\psi}^{x}\right\|$ for some constant $c$.

We now turn to the definition of $G_{x}=\left\langle N_{x}, \Sigma_{x}, R_{x}, A_{x}\right\rangle$. For each variable $y$ of $\psi$ we introduce a nonterminal $A_{y}$. The set $\Sigma_{x}$ contains a terminal symbol $a_{y}$ for each variable $y$ that is universal and later than $x$. If $x$ is universal, then there is also a terminal $a_{y}$ for each variable $y$ that is earlier than $x$. Further, for each clause of the form $\left(x_{1} \leq x_{2} \vee x_{1} \leq x_{3}\right)$ we have a rule $A_{x_{1}} \rightarrow A_{x_{2}} A_{x_{3}}$. For each terminal symbol $a_{y}$ in $\Sigma_{x}$ there is also a rule $A_{y} \rightarrow a_{y}$. It is clear, that such a reduction may be provided using logarithmic space.

Now, if $\mathcal{L}\left(G_{\psi}^{x}\right)$ contains a word $a_{x_{1}} \ldots a_{x_{k}}$, then, by a simple induction, we can show that a clause $\left(x \leq x_{1} \vee \ldots \vee x \leq x_{k}\right)$ belongs to TClosure $(\phi)$. Since each $x_{i}$ for $1 \leq i \leq k$ is universal and later than $x$ or provided $x$ is universal, earlier than $x$; this clause is ultimate. Similarly, if any ultimate clause $\left(x \leq x_{1} \vee \ldots \vee x \leq x_{k}\right)$ belongs to TClosure $(\phi)$, then we can construct a parse tree that witnesses $a_{x_{1}} \ldots a_{x_{k}} \in \mathcal{L}\left(G_{\psi}^{x}\right)$

(About Hardness) The hardness proof is quite similar. This time we give a logspace reduction from the emptiness problem to the problem $\operatorname{QCSP}\left(x_{1} \leq x_{2} \vee x_{1} \leq x_{3}\right)$.

Acknowledgements We thank Jerzy Marcinkowski for turning our attention to [11].

\section{References}

1. Feder, T., Vardi, M.: Monotone monadic SNP and constraint satisfaction. In: Proceedings of 25th ACM Symposium on the Theory of Computing (STOC). (1993) 612-622

2. Schaefer, T.: The complexity of satisfiability problems. In: Proceedings 10th ACM Symposium on Theory of Computing, STOC'78. (1978) 216-226 
3. Jeavons, P., Cohen, D., Gyssens, M.: Closure properties of constraints. Journal of the ACM 44 (1997) 527-548

4. Bulatov, A.: A dichotomy theorem for constraints on a three-element set. In: Proceedings 43rd IEEE Symposium on Foundations of Computer Science (FOCS'02), Vancouver, Canada (2002) 649-658

5. Cohen, D., Jeavons, P.: The complexity of constraints languages. In Rossi, F., van Beek, P., Walsh, T., eds.: Handbook of Constraint Programming. Elsevier (2006)

6. Allen, J.F.: Maintaining knowledge about temporal intervals. Commun. ACM 26(11) (1983) 832-843

7. Krokhin, A., Jeavons, P., Jonsson, P.: A complete classification of complexity in Allens algebra in the presence of a non-trivial basic relation. In: Proceedings of the 17th International Joint Conference on Artificial Intelligence (IJCAI 2001), Morgan Kaufmann (2001) 83-88

8. Bodirsky, M.: Constraint Satisfaction Problems with Infinite Domains. PhD thesis, Humboldt-Universität zu Berlin (2004) http://www2.informatik.hu-berlin. de/ bodirsky/publications/diss.html.

9. Boerner, F., Bulatov, A., Jeavons, P., Krokhin, A.: Quantified constraints: Algorithms and complexity. In: Proceedings of Computer Science Logic and the 8th Kurt Gödel Colloquium. Volume 2803 of Lecture Notes in Computer Science., Springer (2003) 58-70

10. Bodirsky, M., Kára, J.: The complexity of equality constraint languages. In: Computer Science - Theory and Applications, First International Computer Science Symposium in Russia, CSR 2006, St. Petersburg, Russia, June 8-12, 2006, Proceedings. Lecture Notes in Computer Science, Springer (2006) 114-126

11. Bodirsky, M., Chen, H.: Quantified equality constraints. In: 22nd IEEE Symposium on Logic in Computer Science (LICS 2007), 10-12 July 2007, Wroclaw, Poland, Proceedings, IEEE Computer Society (2007) 203-212

12. Bodirsky, M., Kára, J.: The complexity of temporal constraint satisfaction problems. In Ladner, R.E., Dwork, C., eds.: Proceedings of the 40th Annual ACM Symposium on Theory of Computing, Victoria, British Columbia, Canada, May 17-20, 2008, ACM (2008) 29-38

13. Charatonik, W., Wrona, M.: Tractable positive quantified constraint satisfaction problems. Submitted. http://www.ii.uni.wroc.pl/ mwrona/publications/ $\mathrm{TQP} \cdot \mathrm{pdf}(2008)$

14. Bodirsky, M., Chen, H.: Qualitative temporal and spatial reasoning revisited. In: Computer Science Logic, 21st International Workshop, CSL 2007, 16th Annual Conference of the EACSL, Lausanne, Switzerland, September 11-15, 2007, Proceedings. Lecture Notes in Computer Science, Springer (2007) 194-207

15. Möhring, R.H., Skutella, M., Stork, F.: Scheduling with and/or precedence constraints. SIAM J. Comput. 33(2) (2004) 393-415

16. Hodges, W.: A Shorter Model Theory. Cambridge University Press (1997) 